\title{
Nutzerzentrierte Entwicklung des Bediendialoges für die Münchner Fahrkartenautomaten
}

\author{
Fahrkartenautomat_Bediendialog_nutzerzentrierte Entwicklung_Fokusgruppe_Nutzertests_Prototypenentwicklung
}

\begin{abstract}
Fahrkartenautomaten und Kunden - eine Geschichte voller Missverständnisse. Wie oft ist es Ihnen schon so ergangen, dass Sie an einem Automaten ein Ticket kaufen wollten und entweder gar nicht zum Ziel kamen oder nach langem Hin und Her immer noch nicht so recht sicher waren, das richtige Ticket erhalten zu haben. Genau das wollten wir ändern. Unser Ziel war es, Kunden das Gefühl zu vermitteln, vom Automaten verstanden zu werden, und nicht
\end{abstract}

Verständnis für die Eigenheiten des Automaten aufbringen zu müssen. Der vorliegende Beitrag stellt am Beispiel der Entwicklung des Bediendialoges für die stationären Fahrkartenautomaten der Münchner Verkehrsgesellschaft (MVG) vor, wie eine nutzerzentrierte Entwicklung der Bedienabläufe an einem digitalen Kommunikationsinstrument wie einem Ticketautomaten funktionieren kann.

\section{Auftraggeber und Projektziele}

Die Stadtwerke München GmbH (SWM) ist aus dem Alltag der meisten Münchner nicht mehr wegzudenken. Sie ist einer der größten deutschen kommunalen Dienstleister mit verschiedenen Tochtergesellschaften. Eine dieser Tochtergesellschaften ist die Münchner Verkehrsgesellschaft mbH (MVG), die im Auftrag der Stadt München den städtischen U-Bahn-, Straßenbahn- und Stadtbusverkehr in München betreibt. Als zweitgrößtes kommunales Verkehrsunternehmen in Deutschland befördert die MVG jedes Jahr rund 500 Millionen Fahrgäste.

Das komplexe über viele Jahre gewachsene Tarifsystem macht es den Kunden nicht einfach, genau den für sie richtigen Tarif zu wählen. Im Rahmen einer umfangreichen Ersatzbeschaffung plante die MVG den Kauf von 292 neuen Fahrkartenautomaten, um das vorhandene Automatennetz zu modernisieren. Die bisher verwendeten Tastenautomaten werden nach und nach durch Automaten mit Touchscreenmonitor ersetzt. Die neuen Geräte sollten dem Kunden unter anderem folgende Möglichkeiten bieten:

- wie gewohnt seine Fahrkarten zu kaufen, nun aber über die Touch-DisplayOberfläche des Automaten.

DOI 10.1524/icom.2012.0027
- sich per Easy-Taste einen stark vergröBerten Bildschirm anzeigen zu lassen.

- Tickets über einen Barcode-Scanner auszuwählen und erhöhtes Beförderungsentgelt am Automaten einzuzahlen. Alle Tickets und Einzahlungsbelege für erhöhtes Beförderungsentgelt werden künftig mit einem QR Code ausgestattet sein. Scannt der Nutzer diesen QR Code am Automaten, gelangt er bei einem Ticket direkt in den Warenkorb und bei einem Beleg für erhöhtes Beförderungsentgelt direkt in den dazu passenden Eingabemodus.

- mit höherwertigen Banknoten zu zahlen und Wechselgeld in Scheinform zu erhalten. Je nach Art des Tickets kann der Kunde also auch mit einem 50 oder 100 Euroschein sein Ticket bezahlen.

Zudem können die Kunden bargeldlos per EC-, Geld- und Kreditkarte zahlen.

Im Sommer 2009 beauftragte die Stadtwerke München GmbH die HANDSPIEL GmbH aus Leipzig, die Bedienabläufe des neuen Kundendialoges der neuen Fahrkartenautomaten unabhängig von der parallel laufenden technischen Ausschreibung der Hardware zu erarbeiten. Die SWM verband mit der Einführung der neuen Automaten verschiedene Erwartungen. Den Kunden sollte es einfacher gemacht werden, schnell und unkompliziert zu „ihren" Fahrkarten zu kommen.
Kunden, die bisher Schwierigkeiten mit den Automaten hatten, sollte die Bedienung spürbar erleichtert werden. Die schnellere Bedienabwicklung an den Geräten sollte die einzelnen Verkaufsvorgänge an den Geräten verringern und Wartezeiten minimieren. Zudem sollten Fehlkäufe verringert werden, die Folgekosten verursachen

\section{Nutzerzentrierte Entwicklung}

Grundsätzlich ist die Einführung eines neuen Fahrkartenautomaten bei einem Verkehrsanbieter mit einer Produkteinführung gleichzusetzen. Produkteinführungen bergen sowohl hohe Risiken als auch große Chancen. Ein Fahrkartenautomat kostet, je nach Hersteller, zwischen 20.000 und 50.000 Euro. Bei der Anschaffung 292 neuer Geräte sollte das Risiko einer teuren Fehlinvestition minimiert werden. So können Mängel in der Informationsarchitektur eines Fahrkartenautomaten erhebliche Umsatzeinbußen und Imageverluste für das Verkehrsunternehmen verursachen. Eine funktionelle Gestaltung kann nicht nur zu einer Imageverbesserung führen, sondern auch zu einer verstärkten Nutzung der Automaten.

Nicht nur die technische Realisation der gewünschten Funktionalitäten, auch 
die Gestaltung des digitalen Dialogs zwischen Kunde und Fahrkartenautomat stellt somit ein zentrales Thema dar. Treten erst nach dem Roll-out Nutzerprobleme an den Geräten zutage, lassen sich diese nur mit viel Zeit- und Kostenaufwand korrigieren. Aus diesem Grund war es der MVG wichtig, die künftigen Nutzer so früh wie möglich in die Entwicklung der Bediendialoge einzubeziehen und deren Wünsche und Erwartungen in dem Entwicklungsprozess ausreichend zu berücksichtigen.

Dieser Aufgabe näherte sich das hier vorgestellte Projekt mit einer Kombination verschiedener Usability-Methoden, nämlich Use-Cases, Personas, einer Fokusgruppe sowie Software-Prototyping in Verbindung mit iterativen Nutzertests (Usability-Rapid-Prototyping).

\subsection{Use Cases und Persona- Entwicklung}

Zu Beginn des Projektes stand eine eingehende Analyse der projektspezifischen Ausgangsbedingungen. Neben der intensiven Auseinandersetzung mit dem Münchner Tarifsystem standen die Eruierung bestehender Nutzerprobleme und die Sichtung einschlägiger Gestaltungsrichtlinien im Mittelpunkt der Aufmerksamkeit des Projektteams, wie beispielsweise verschiedene ISO-Normen zur Usability, die DIN 32975 zur Gestaltung visueller Informationen im öffentlichen Raum zur barrierefreien Nutzung, sowie die Empfehlungen der Schweizerischen Fachstelle für Behinderte und öffentlichen Verkehr. Das Projektteam bestand auf Seiten von HANDSPIEL aus einer Projektmanagerin, zwei Konzeptionern, einer Grafikerin, einem Programmierer und einer Wirtschaftspsychologin. Aufseiten der SWM arbeiteten ein Projektmanager und sukzessive Kollegen aus den Fachabteilungen Automatenwerkstatt, Vertrieb, Marketing und Marktforschung an dem Projekt mit.

Neben der Literatur- und Internetrecherche nutzte das Team das von der SWM zur Verfügung gestellte Datenmaterial, um sich mit den Rahmenbedingungen des Projektes vertraut zu machen. Zu den zur Verfügung gestellten Materialien gehörten beispielsweise interne Kundenfeedbackprotokolle und Ticketverkaufszahlen zu den einzelnen
Ticketkategorien. In dieser Projektphase entstanden auch Use Cases und Personas zu den verschiedenen Nutzergruppen des Ticketautomaten. Für die Entwicklung der Personas wurde unter anderem auf die Sinus-Milieus in Bezug auf Mobilität zurückgegriffen. Die Nutzung des ÖPNV ist in den unterschiedlichen Sinus-Milieus unterschiedlich stark ausgeprägt. Auch die Haltung gegenüber dem ÖPNV ist unterschiedlich, je nachdem ob die Nutzer aus bewusster Entscheidung oder aus finanziellem Zwang den ÖPNV nutzen. Die unterschiedliche Repräsentation der verschiedenen Sinus-Milieus und ihre abweichenden Motive spiegelten sich auch in den entwickelten Personas wieder. Insgesamt entstanden fünf verschiedene Persona-Profile, die dem Projektteam während des gesamten Entwicklungsprozesses halfen, konzeptionelle Entscheidungen zu bewerten und im Sinne der Nutzer zu treffen. Die Use Cases bildeten verschiedene Anwendungsfälle $a b$ wie beispielsweise den Kauf verschiedener Ticketarten auf Basis der Personas, die Einzahlung von erhöhtem Beförderungsentgelt oder auch die Nutzung des Barcodescanners.

\subsection{Konzeptentwicklung und Fokusgruppe}

Auf Basis der Status-quo-Analyse, der Personas und Use Cases entstanden erste Ablaufskizzen für die Bedienoberfläche an den Geräten. Diese wurden in einem ersten Softwareprototypen umgesetzt. Die Konzeptentwürfe und der erste Softwareprototyp wurden in einer Fokusgruppe diskutiert. Für diese erste Konfrontation wurden ÖPNV-Kunden im Alter zwischen 63 und 73 Jahren rekrutiert, da die Einführung einer Neuerung wie Touch-Displays insbesondere für ältere Nutzer eine Herausforderung darstellen könnte, die wenig oder keine Vorerfahrung mit dieser Technologie mitbringen. Daher war es von besonderem Interesse, Bedürfnisse und mögliche Nutzungsprobleme dieser Personengruppe kennenzulernen.

Die Teilnehmer (5 Frauen, 4 Männer) lebten seit durchschnittlich 38 Jahren in München. Die meisten Frauen nutzten den ÖPNV bereits während ihrer Berufstätigkeit, während die Männer erst nach ihrer Pensionierung auf den ÖPNV zurückgriffen. Die Teilnehmer gaben an, dass sie sowohl Verkaufsstellen als auch Ticketautomaten für den Fahrkartenkauf nutzen. Dabei fallen ihnen gerade Routinekäufe an den Automaten leicht: "Ich kaufe meist eine Partnertageskarte. Da weiß ich auch ohne Brille, welche Taste ich drücken muss. Die vierte Taste von oben rechts." (Herr S.). Müssen sie jedoch von ihren Fahrtroutinen abweichen oder werden sie von Ortsunkundigen angesprochen, kommen sie häufig in Verlegenheit: "Ich werde häufig mal vor den Automaten angesprochen, ob ich vielleicht helfen könnte und dann steh ich auch wie der Ochs vorm Berg." (Herr H.)

Die Fokusgruppenteilnehmer wünschten sich

- eine Beschränkung auf wesentliche Inhalte bei der Informationsdarbietung,

- einen extra Bereich, in dem Sehenswürdigkeiten und häufig gesuchte Ziele (z.B. Flughafen, Messe) hinterlegt sind,

- größere und gut lesbare Schriften und - den Einsatz von Piktogrammen, um fremdsprachigen und Nutzern mit einer Leseschwäche die Orientierung am Automaten zu erleichtern.

Zudem wünschten sie sich eine bessere Erklärung von Zonen und Ringen. Aufgrund der Komplexität des Tarifsystems und seiner regionalgeprägten Begrifflichkeiten wünschten sie sich hier grafische Übersetzungshilfen und eine stärkere Nutzerführung, durch das System.

\subsection{Usability-Rapid- Prototyping}

Auf Basis der Anregungen und Optimierungsvorschläge aus der Fokusgruppe wurde der Software-Prototyp angepasst und es entstand das erste Screendesign für die Bedienerführung. Parallel dazu wurde von einem Messebauer ein 1:1-Dummy des später einzusetzenden Fahrkartenautomaten gebaut. Mithilfe der Originalmaße des Automaten, den Automatenoriginalteilen (z. B. Ausgabeschale und PIN-Pad) des Herstellers sowie einem Touchscreenmonitor ähnelte er zum Verwechseln den späteren „echten“ Automaten des Herstellers ICA (siehe Bild 1). Zur Steuerung der Testszenarien und der Messung der Bedienzeiten sowie Aufzeichnung erforderlicher Korrek- 
turschritte des Probanden während der Nutzertests, wurde ein eigens entwickelter Steuerungsmonitor verwendet. Über diesen Steuerungsmonitor ließ sich beispielsweise der Fahrkartenkauf mit unterschiedlichen Zahlungsmitteln simulieren.

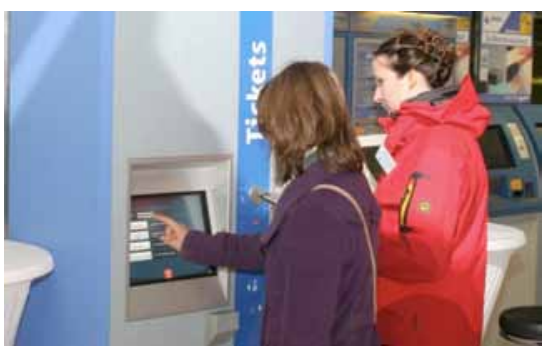

Bild 1: Nutzertest an einem Münchner U-Bahnhof

Im Rahmen des Usability-Rapid-Prototyping wurden insgesamt fünf Nutzertests an verschiedenen U-Bahnhöfen in München durchgeführt. Im Mittelpunkt der Tests standen jeweils unterschiedliche Fragestellungen und Nutzergruppen. So gab es separate Nutzertests mit einem Schwerpunkt auf ältere Nutzer, Mobilitätseingeschränkte und fremdsprachige Touristen. Ein wesentlicher Vorteil des Softwaredummys war es, dass an ihm Änderungen schnell und unkompliziert vorgenommen werden konnten. Dadurch war es möglich, die verschiedenen grafischen und inhaltlichen Elemente der Bedienoberfläche immer wieder auf ihre anvisierte Wirkung bzw. Akzeptanz bei den Kunden zu testen und falls erforderlich bereits für den nächsten Nutzertest zu überarbeiten.

Ziel war es, den Kundendialog so zu strukturieren, dass die späteren Nutzer die gesuchten Informationen an den Stellen vorfinden, an denen sie diese intuitiv erwarten. Dabei spielen verschiedene Kriterien eine Rolle:

- die Seiten müssen übersichtlich aufgebaut sein,

- die dargebotenen Informationen dürfen die Nutzer nicht überfordern,

- die Nutzer müssen eine Struktur erkennen und

- die Navigationselemente müssen als solche erkannt und verstanden werden.

Für die Nutzertests wurde ein Untersuchungskonzept erarbeitet, das aus Simulationsaufgaben und strukturierten Interviewfragen bestand. Dadurch konnten in den Interviews sowohl die Verständlichkeit der einzelnen Bedienabläufe als auch einzelne Aspekte der Gestaltung und die Erfüllung der europäischen ISO-Normen abgeprüft werden.

Der erste Nutzertest fand in der UBahnstation am Marienplatz in München statt. Er diente der Eruierung der bis dahin entstandenen Nutzerführung, also der Überprüfung, welche Elemente und Bedienwege des Kundendialogs bereits gut verständlich waren und an welchen Punkten es zu Missverständnissen oder Nutzungsbarrieren kam.

Eine erste zentrale Nutzungsbarriere war beispielsweise der Bildschirmschoner. Die Buttons des Startbildschirms wurden auf dem zweigeteilten Bildschirmschoner abgedunkelt dargestellt. Über diesem Buttonbereich kreiste eine stilisierte Hand, die die Nutzer zur Deaktivierung des Bildschirmschoners auffordern sollte. Doch gerade dieser Bildschirmschoner irritierte einige Probanden. Einige wählten bereits einen Ticketbereich aus (z.B. „Einzelfahrkarten \& Streifenkarten") und waren daraufhin irritiert, dass sie diese Auswahl nach der Deaktivierung des Bildschirmschoners erneut treffen mussten.

Bei den anschließenden vier weiteren Nutzertests traten einige Schwierigkeiten trotz grafischer Modifikationen erneut auf (z.B. Bildschirmschoner). Andere Nutzerschwierigkeiten dagegen konnten bereits nach dem ersten Test behoben werden. Mit der grafischen und funktionalen Weiterentwicklung des Prototy- pen stellten sich jedoch auch neue Nutzerschwierigkeiten ein, die beim ersten Test noch nicht in Erscheinung traten. So verursachte beispielsweise der Barcodescanner erhebliche Nutzerprobleme. Die Verständnisprobleme gingen dabei sogar so weit, dass einige der Probanden die Aufforderung auf dem Monitor „Barcode jetzt scannen!" wortwörtlich nahmen und den Barcode zum scannen auf den Monitor auflegten. Durch grafische Änderungen sowohl im Kundendialog als auch der Außenbeschriftung konnte dieses Problem jedoch gelöst werden. Weitere Verständnisprobleme betrafen einzelne Navigationselemente im Kundendialog wie beispielsweise die Reiternavigation (siehe Bild 2). Hier war die Navigation über Reiter nicht für alle Nutzer verständlich. Diese Problematik wurde durch einen gesonderten „Blätter-Button“ gelöst, nachdem grafische Anpassungen hinsichtlich Anordnung und Farbgestaltung nicht zum gewünschten Ergebnis führten. Der „Blätter-Button“ ermöglicht es den Nutzern, zwischen den Kategorien zu wechseln, auch ohne die Reiternavigation zu nutzen (siehe Bild 3).

Insgesamt lieferten die fünf Nutzertests wertvolle Anregungen für die Gestaltung des Kundendialoges. Jeder einzelne Nutzertest lieferte neue Erkenntnisse und zugleich eine Rückmeldung darüber, ob die grafischen und konzeptio-

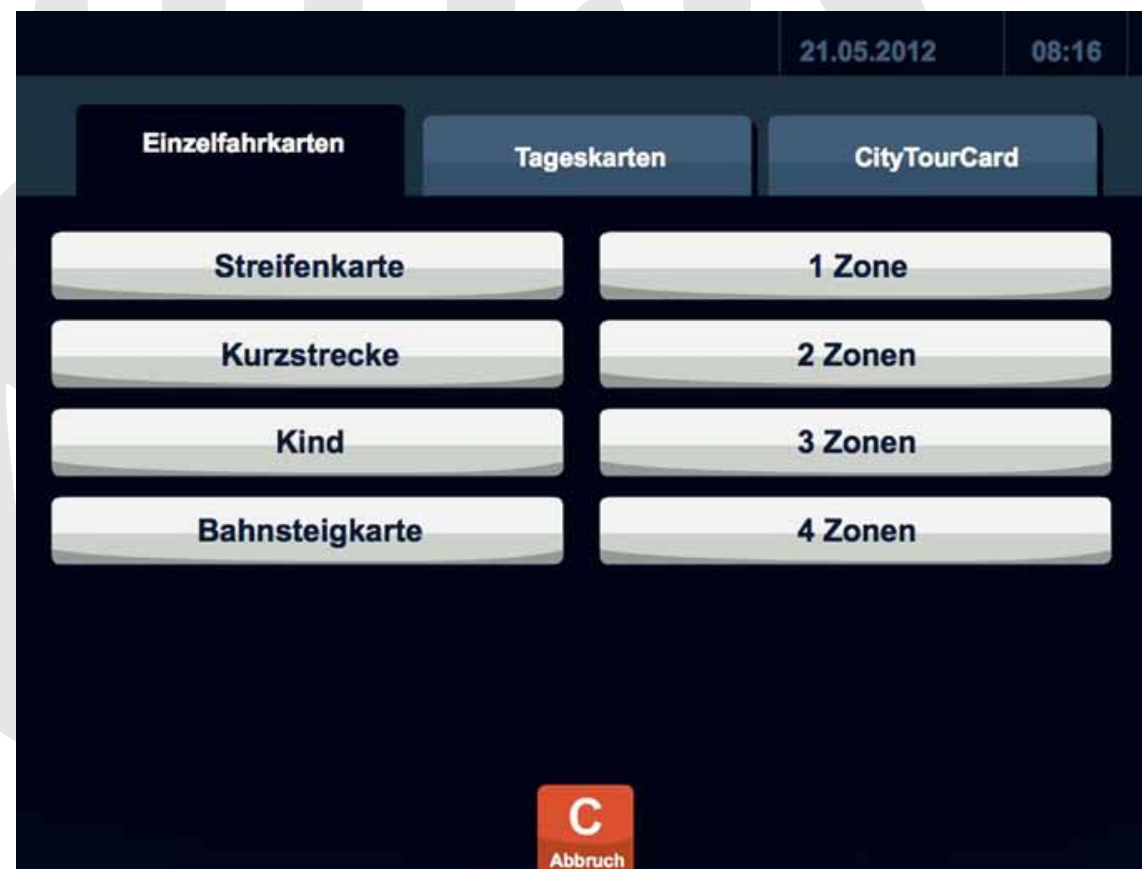

Bild 2: Schnellwahl ohne Weiter-Button 


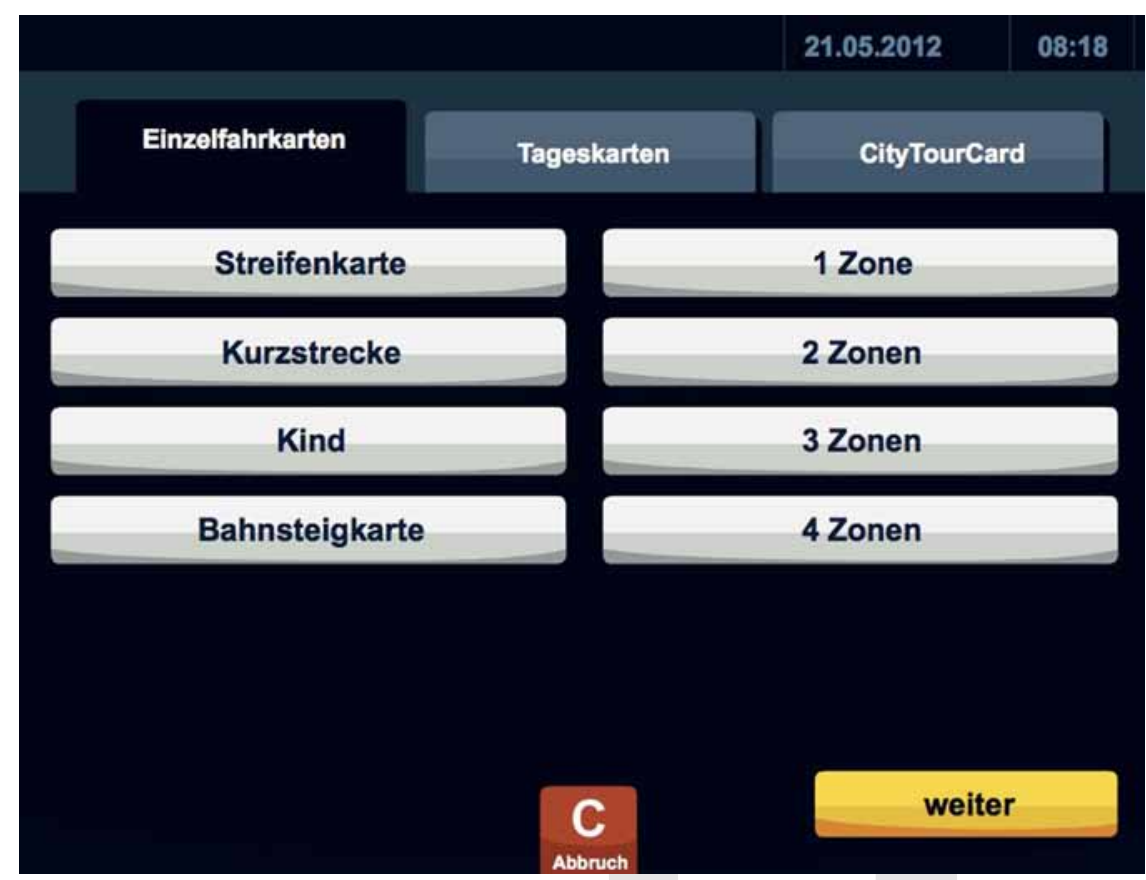

Bild 3: Schnellwahl mit Weiter-Button

nellen Änderungen an dem Bediendialog für das Nutzerverständnis sinnvoll waren.

Nach Abschluss der Nutzertests wurden das entwickelte Bedienkonzept und das Screendesign dokumentiert und dem Automatenhersteller zur technischen Umsetzung an den Geräten übergeben. Zum Abschluss des Projektes erfolgte eine Qualitätssicherung an den Fahrkartenautomaten, um die Umsetzungsqualität des Bediendialoges an den Geräten sicher zu stellen.

\section{Resonanz}

Die gewählte Vorgehensweise bei der Konzeption des neuen Kundendialoges fand sowohl bei der SWM als auch dem Automatenhersteller ICA und den getesteten Nutzern eine positive Resonanz. Durch die Zusammenarbeit mit der ICA und deren Programmierern konnte sichergestellt werden, dass die gefundene Lösung für den Kundendialog sich technisch problemlos auf den Automaten umsetzen lässt. Bereits während der Nutzertests äuBerten viele der Befragten ihre Zustimmung darüber, „auch mal gefragt zu werden". Auf die geplante Einführungskampagne bei der Hostessen Assistenz beim Kauf des passenden Tickets bieten sollten, konnte kurzerhand verzichtet werden. Bereits in den ersten Stunden zeigte sich, dass Nutzer ganz ohne vorherige Einführung das passende Ticket kaufen konnten. Die neuen Automaten schienen den Kunden tatsächlich das Gefühl vermittelten, verstanden zu werden die nach Änderungen im Bedienkonzept sonst typischen Kundenbeschwerden blieben aus. Erste Fahrgastbeobachtungen ergaben, dass der Kunde keine eindeutige Präferenz für eine bestimmte Automatengeneration hat. Der Touch kann somit mindestens genauso schnell und einfach bedient werden, wie der alt bekannte stationäre Tastenautomat. So bemerkte auch Michael Kalenda (Leiter Verkaufsstellen- und Automatenvertrieb Stadtwerke München $\mathrm{GmbH}$ / Münchner Verkehrsgesellschaft (MVG), dass die neuen Automaten gut angenommen werden: „Ist erstmal die Zugangshürde des Touchbildschirms überwunden, so stellten die Kunden bereits nach wenigen Sekunden fest: „Das ist ja einfach!“.”

Mit dem Aufstellen der neuen Automaten ist die Entwicklung jedoch noch nicht zu Ende. Unabhängig von Änderungen im Verkaufssortiment laufen weiterhin Aktivitäten zum Feintuning der Dialoge. Änderungen am Kundendialog werden per Fernwartung als Update auf die Geräte aufgespielt, wie kürzlich beispielsweise ergänzende Informationen zum Geldwechsler. So flieBen laufend neue Erkenntnisse aus dem Praxisumfeld und Kundenrückmeldungen ein, um die Dialoge noch ein Stück besser zu machen.

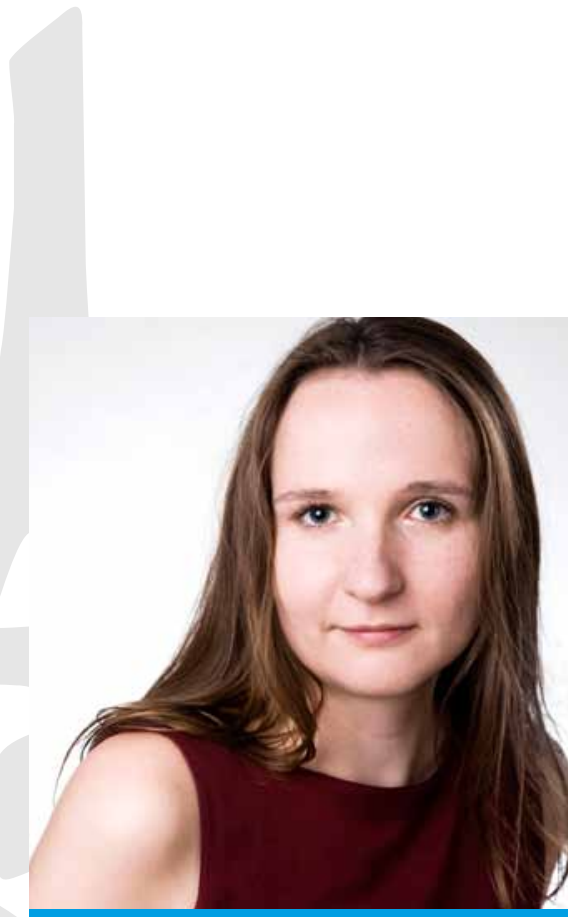

Irka Schneider

ist studierte Wirtschaftspsychologin mit den Schwerpunkten Markt- und Konsumenten-psychologie sowie Ingenieurspsychologie. Nach ihrem Studienabschluss arbeitete sie zunächst freiberuflich als Usabilityberaterin für verschiedene Projekte. Seit 2007 leitet sie den Usability-Bereich bei der HANDSPIEL GmbH und setzte zusammen mit ihrem Team nutzerorientierte Softwarekonzeptionsprojekte um. Dabei begleitet sie den Entwicklungsprozess von den ersten Vorgesprächen mit den Auftraggebern über die Konzeptions- und Entwicklungsphase bis zum finalen Roll-out der Produkte. E-Mail: schneider@handspiel.net 